\title{
Editorial
}

Z Herz- Thorax- Gefäßchir 2020 · 34:277-279 https://doi.org/10.1007/s00398-020-00391-1

(C) Springer Medizin Verlag GmbH, ein Teil von Springer Nature 2020

\section{Jochen Cremer}

Klinik für Herz- und Gefäßchirurgie, Universitätsklinikum Schleswig-Holstein, Campus Kiel, Kiel, Deutschland

\section{Herzchirurgie in der Hitzewelle}

Liebe Leserinnen, liebe Leser,

unter den Isolations- und Reklusionsbedingungen in Coronazeiten hat sich das herzchirurgische Leben, mal ganz abgesehen von den Operationsspektren und Tagesabläufen in den Kliniken, offensichtlich tiefgreifend geändert. Irgendwie besteht der Eindruck, als gäbe es weniger aktuelle kritische Diskussionen und Publikationen und auch weniger berufspolitische Kontroversen, sodass die aktuelle Situation wirkt wie ein fachlicher „shut-down“ mit exklusiver Umfokussierung auf Coronathemen: also weniger „hot tops“, aber mehr „hot spots“.

Dazu kommt dann noch, dass wir in Schleswig-Holstein (um auf die lokale Situation einmal umzuschwenken) seit mehr als 2 Wochen eine substanzielle Hitzewelle haben, die zwar hier den Tourismus enorm beflügelt, aber sich lähmend auswirkt, auf geistige Versatilität, Ideenblitze und produktives „output“.

Stellt man sich die Frage, ob diese Hitzewelle möglicherweise auch Effekte auf das routinierte klinische Arbeiten und Veränderungen für den Behandlungsverlauf der unter diesen Bedingungen operierten Patienten mit sich bringt, findet man dann unter Benutzung der etablierten Suchmaschinen eine Reihe interessanter Aspekte und Fakten sowohl für generell chirurgische Eingriffe als auch herzchirurgische Prozeduren im Speziellen.

So wird in folgenden Statements u. a. exemplarisch darauf hingewiesen, dass:

1. „Hot and humid weather adversely affects perioperative outcome in elderly surgical patients." (Gautam) [1]

2. „Seasonal variations in CHF-related morbidity and mortality occur in hot climate..." (Inglis) [2]
3. „At population level, SSI [surgical site infection] risk is highly seasonal and associated with warmer weather."

(Anthony) [3]

Dies sind nur einzelne Passagen, die zugegebenermaßen sehr punktuell herausgegriffen wurden; nur lässt sich auch definitiv feststellen, dass es um die "heat shock proteins" bei herzchirurgischen Eingriffen offenbar sehr ruhig geworden ist.

Kommen wir noch einmal zurück auf die individuellen Bedingungen im herzchirurgischen Alltag, so kann man diesen Zustand für die nördlichste deutsche Herzchirurgie hier in Kiel ungefähr so beschreiben:

Die Patientenzimmer mit Jalousien und "shutters" vermitteln von Raumund Ausstattungsgefühl ein gehobenes mediterranes Ambiente, das sich von der mittleren Kühle und Funktionalität in

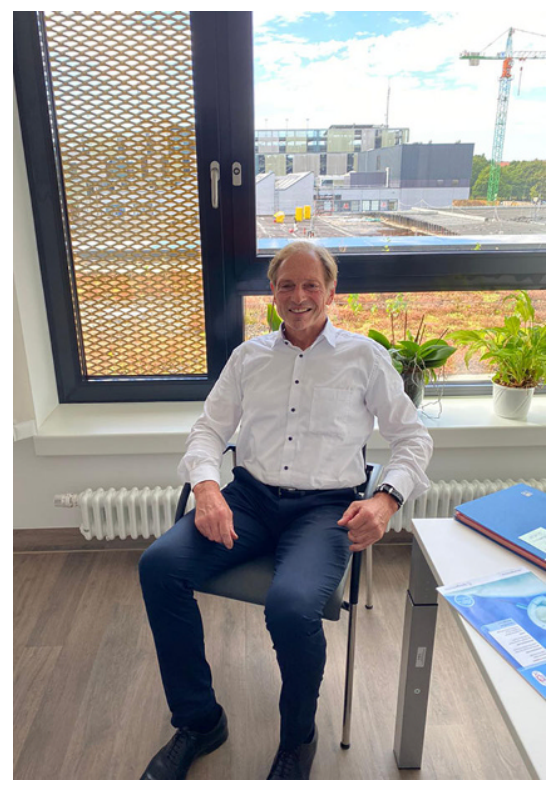

Abb. $1 \Delta$ Die Hitzewelle spart auch den OfficeBereich des UKSH am Campus Kiel nicht aus 
Hier steht eine Anzeige.

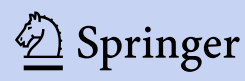


„intermediate care“ und Intensivmedizin positiv abhebt.

Die Temperaturbetroffenheit der Mitarbeiterinnen und Mitarbeiter in den verschiedenen $\mathrm{OP}$ ist recht unterschiedlich; im Extremfall kann das auch mal bedeuten: $17^{\circ} \mathrm{C}$ eingestellt an der Klimaanlagensteuerung, $21,6^{\circ} \mathrm{C}$ Messwert im Display bei einem Raumluftmesswert $>27^{\circ} \mathrm{C}$. Dies wird dann noch übertroffen im Chefarzt-Office (sei klug, werd' Chef) mit Temperaturen über $35^{\circ} \mathrm{C}$, die durch die sommerliche Thermik der direkt angrenzenden großflächigen Lavasteinterrasse auch noch Potenzial für heißen Aufwind und damit weitere Steigerungen hat. Zumindest für den Sommer gilt somit: In der Herzchirurgie im nördlichsten Norden herrscht kein raues Klima (•Abb. 1).

Resümee: Neben Corona gibt es sicherlich eine ganze Reihe von weiteren heißen Themen, von denen eines ganz sicher sein könnte: ,die klimatischen Bedingungen und ihre Effekte in der Herzchirurgie“.

Der eine oder andere mag sich angesprochen und inspiriert fühlen, in diesem Themenkomplex einmal wissenschaftlich vorzugehen. Wenn eine Aussage dabei wäre, Herzchirurgie muss ganz unbedingt bei Temperaturen zwischen 18 und $22^{\circ} \mathrm{C}$ stattfinden, hätte dies sicherlich genug Power, das Hygieneteam, die Geschäftsführung und den Klinikumsvorstand auf den Plan zu rufen, um das Klinikumsklima chirurginnen-, chirurgen- und chirurgiefreundlich $\mathrm{zu}$ gestalten.

Wer mir weiterführende sachdienliche Informationen liefert, kann - einmal vorsichtig formuliert - u. U. mindestens mit einer großen Kugel Eis rechnen.

Ihr

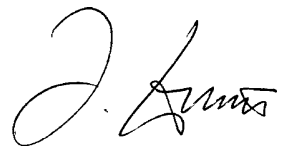

Jochen Cremer

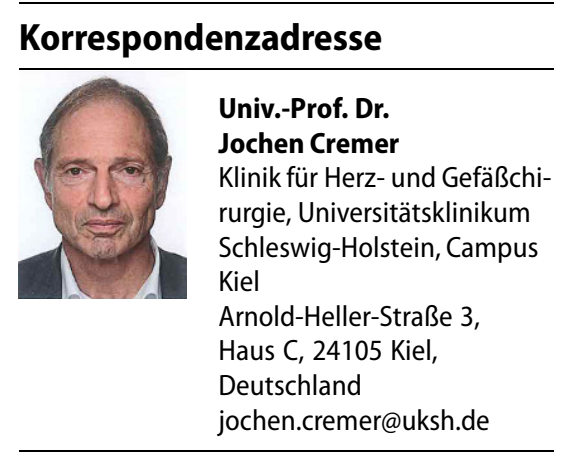

Interessenkonflikt. J. Cremer gibt an, dass kein Interessenkonflikt besteht.

\section{Literatur}

1. Gautam PL, Kathuria S, Chhabra S (2011) Hot climate and perioperative outcome in elderly patients. Indian J Crit Care Med 15(2):88-95

2. Inglis SC, Clark RA, Shakib S, Wong DT, Molaee P, Wilkinson D, Stewart S (2008) Hot summers and heart failure: seasonal variations in morbidity and mortality in Australian heart failure patients (1994-2005). Eur J Heart Fail 10:540-549

3. Anthony CA, Peterson RA, Polgreen LA, Sewell DK, Polgreen PM (2017) The seasonal variability in surgical site infections and the association with warmer weather: a population-based investigation. Infect Control Hosp Epidemiol 38(7):809-816
Aktuelle Informationen

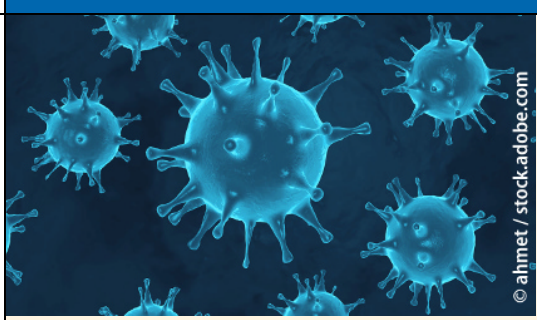

\section{Aktuelle Entwicklung zu COVID-19 bei Springer Nature und Springer Medizin}

Springer Nature und Springer Medizin unterstützen die globale Reaktion auf die COVID-19-Pandemie, indem ein schneller und direkter $\mathrm{Zu}$ gang zu den neuesten verfügbaren Forschungsergebnissen und Daten ermöglicht wird.

Auf der Homepage SpringerMedizin.de finden Sie ein immer aktuelles Dossier mit Beiträgen, Forschungsarbeiten und Ergebnissen zu SARS-CoV-2 sowie relevanten Links.

Darin z.B. auch die kürzlich publizierte

Empfehlung von DIVI, DGIIN, DGAI und DGP zur Intensivtherapie von Patienten mit COVID-19.

Springer Nature arbeitet mit globalen Organisationen zusammen, und verlinkt über SpringerNature.com/de auf eine eigene Landingpage mit einer Vielzahl an Information sowie freiem Zugriff auf die COVID-19-Contentplattformen von Nature Research, BioMed Central (BMC) und Springer.

Das Dossier zu Coronavirus / Covid-19 von Springer Medizin finden Sie hier: www.springermedizin.de/covid-19

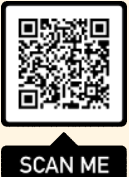

